\title{
Brownian Motion in an External Field Revisited
}

\author{
Angelo Plastino $^{1,2}$, Mario Carlos Rocca ${ }^{1,3,4}$, Diana Monteoliva ${ }^{1,5}$, Alberto Hernando ${ }^{2}$ \\ ${ }^{1}$ Departamento de Física, Universidad Nacional de La Plata, La Plata, Argentina \\ ${ }^{2}$ Kido Dynamics SA, Lausanne, Switzerland \\ ${ }^{3}$ Departamento de Mateática, Universidad Nacional de La Plata, La Plata, Argentina \\ ${ }^{4}$ Consejo Nacional de Investigaciones Científicas y Tecnológicas, (IFLP-CCT-CONICET)-C. C. 727, La Plata, Argentina \\ ${ }^{5}$ Comisión de Investigaciones Científicas, Provincia de Buenos Aires La Plata, La Plata, Argentina \\ Email: angeloplastino@gmail.com
}

How to cite this paper: Plastino, A., Rocca, M.C., Monteoliva, D. and Hernando, A. (2021) Brownian Motion in an External Field Revisited. Journal of Modern Physics, 12, 82-90.

https://doi.org/10.4236/jmp.2021.122008

Received: December 1, 2020

Accepted: January 15, 2021

Published: January 18, 2021

Copyright (c) 2021 by author(s) and Scientific Research Publishing Inc. This work is licensed under the Creative Commons Attribution International License (CC BY 4.0).

http://creativecommons.org/licenses/by/4.0/

\begin{abstract}
In many interesting physical examples, the partition function is divergent, as first pointed out in 1924 by Fermi (for the hydrogen-atom case). Thus, the usual toolbox of statistical mechanics becomes unavailable, notwithstanding the well-known fact that the pertinent system may appear to be in a thermal steady state. We tackle and overcome these difficulties hereby appeal to firmly established but not too well-known mathematical recipes and obtain finite values for a typical divergent partition function, that of a Brownian particle in an external field. This allows not only for calculating thermodynamic observables of interest, but for also instantiating other kinds of statistical mechanics' novelties.
\end{abstract}

\section{Keywords}

Divergent Partition Functions, Statistical Mechanics, Fisher Information

\section{Introduction}

In many interesting physical examples, the partition function is divergent [1] [2] [3] [4]. Thus, the usual toolbox of statistical mechanics becomes unavailable, notwithstanding the well-known fact that the pertinent system may appear to be in a thermal steady state (see, for instance [5] [6] [7] [8] [9]) and references therein]. Our goal here is to deal with a specific divergent partition function, and obtain a finite value for it. This permits to compute new observables of interest and also to develop some hopefully new statistical mechanics' insights.

\section{The Central Issue}

\subsection{Partition Function}

We will consider here the partition function for Brownian motion in an external 
field, given by [4]

$$
\mathcal{Z}=\int_{-\infty}^{\infty} \mathrm{e}^{\frac{\beta U_{0}}{1+x^{2}}} \mathrm{~d} x
$$

with $\beta=1 /\left(k_{B} T\right)$ and $k_{B}=$ Boltzmann's constant. Change now variables to $y=1+x^{2}$. Taking advantage now of well-known features of Schwartz' theory of distributions [10], we can recast the integral that defines $\mathcal{Z}$ in the fashion

$$
\mathcal{Z}=\int_{1}^{\infty}(y-1)^{-\frac{1}{2}} \mathrm{e}^{\frac{\beta U_{0}}{y}} \mathrm{~d} y \equiv \lim _{v \rightarrow 1} \int_{1}^{\infty} y^{\nu-1}(y-1)^{-\frac{1}{2}} \mathrm{e}^{\frac{\beta U_{0}}{y}} \mathrm{~d} y,
$$

and remember that the limit of an integral equals the integral of the limit. We consult then the Table of Ref. [11] and find that our current integral is a special case of the more general one

$$
W=\int_{u}^{\infty} x^{\nu-1}(x-u)^{\mu-1} \mathrm{e}^{\frac{\beta}{x}} \mathrm{~d} x=B(1-\mu-v, \mu) u^{\mu+\nu-1} \phi\left(1-\mu-v ; 1-v, \frac{\beta}{u}\right) .
$$

Here $B$ is the well-known beta function and $\phi$ the confluent hypergeometric function, that reads, appealing to the Gamma function $\Gamma$,

$$
B=\Gamma(1-\mu-v) \Gamma(\mu) / \Gamma(1-v) .
$$

Comparing integrals, we see at this stage that the right hand side of (2.2) will coincide with $W$ in (2.3) by setting

$$
\mu=1 / 2 ; v=1 ; u=1,
$$

so that these special values are to be inserted in

$$
W=\Gamma(1-\mu-v)[\Gamma(\mu) / \Gamma(1-v)] u^{\mu+v-1} \phi\left(1-\mu-v ; 1-v, \frac{\beta}{u}\right) .
$$

Note also that

$$
\Gamma(1 / 2)=\sqrt{\pi} ; \Gamma(-1 / 2)=-2 \sqrt{\pi} .
$$

We have a $\Gamma(0)$ in a denominator now. This induces us to appeal once again to [11] to employ the useful relation

$$
\lim _{\gamma \rightarrow 0} \phi(\alpha ; \gamma ; s)=z \alpha \phi(\alpha+1 ; 2 ; z),
$$

so that we can finally arrive at the result

$$
\mathcal{Z}=\pi \beta U_{0} \phi\left(\frac{1}{2} ; 2 ; \beta U_{0}\right),
$$

our desired finite form. We see that we arrive at $\mathcal{Z}$ via a straightforward path. The essential step here is that of consulting an appropriate table of integrals and performing adequate manipulations. Note that at very low temperatures quantum effects raise their head and our treatment becomes invalid. Below it will be shown that one also encounters problems or exceedingly high temperatures. We have found a finite partition function for our Brownian problem and proceed to calculate with it, below, important quantifiers of statistical mechanics. 


\subsection{Units for Our Graphs}

We find it convenient to plot our thermal quantities versus $y=k_{B} T / U_{0}$ in the range $0 \leq y \leq 1$. Given the smallness of $k_{B}$, this encompasses an immense $T$-range, since $k_{B}$ is of the order of $10^{-23}$ in its appropriate units. In particular, we plot the logarithm of the partition function in Figure 1. We appreciate the fact that it converges to a definite value as $T$ grows.

\section{Other Thermal Quantities}

\subsection{Mean Energy}

One has

$$
\langle\mathcal{U}\rangle=-\frac{\partial \ln \mathcal{Z}}{\partial \beta},
$$

so that

$$
\langle\mathcal{U}\rangle=-\frac{1}{\mathcal{Z}}\left[\pi U_{0} \phi\left(\frac{1}{2} ; 2 ; \beta U_{0}\right)+\frac{\pi \beta U_{0}^{2}}{4} \phi\left(\frac{3}{2} ; 3 ; \beta U_{0}\right)\right] .
$$

Note that at very low temperatures quantum effects raise their head and our classical treatment becomes invalid.

\subsection{Entropy $\mathcal{S}$}

We have

$$
S=\frac{\partial\left(k_{B} T \ln Z\right)}{\partial T}
$$

so that

$\mathcal{S}=\ln \left[\pi \beta U_{0} \phi\left(\frac{1}{2} ; 2 ; \beta U_{0}\right)\right]-\frac{\beta}{\mathcal{Z}}\left[\pi U_{0} \phi\left(\frac{1}{2} ; 2 ; \beta U_{0}\right)+\frac{\pi \beta U_{0}^{2}}{4} \phi\left(\frac{3}{2} ; 3 ; \beta U_{0}\right)\right]$,

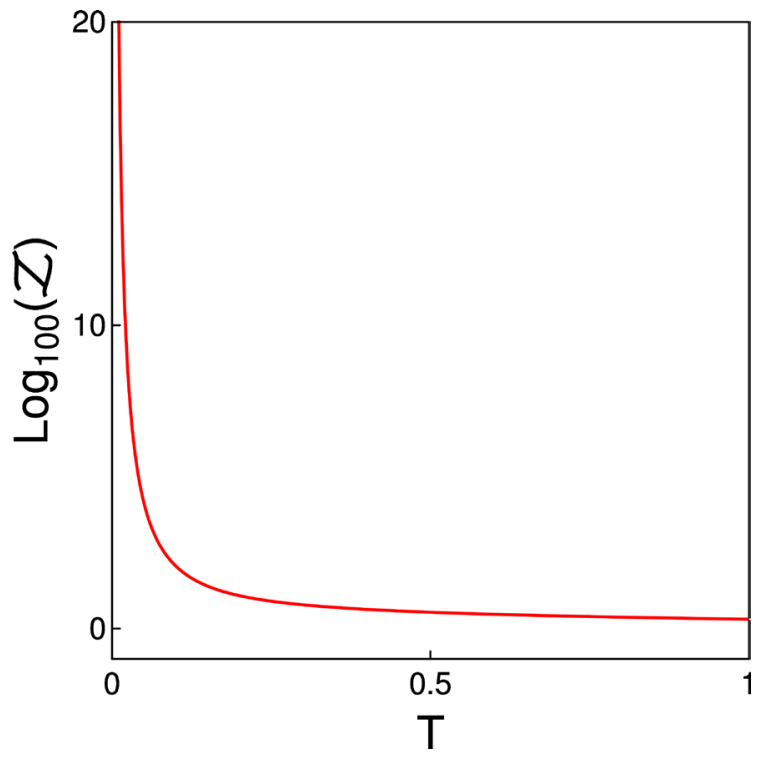

Figure 1. Logarithm of the partition function in appropriate units (see text). 
that is plotted in Figure 2. Note that at very low temperatures, quantum effects raise their head and our treatment becomes invalid. This is evident whenever $S$ becomes negative at low $T$. A new effect is observed at very large $T$. Whenever $T \geq 10^{22}$, the treatment becomes invalid as well. Such high- $T$ outcome is typical of classical self-gravitating systems [12] [13] [14].

\subsection{Specific Heat $\mathcal{C}$}

One defines it as

$$
\mathcal{C}=-\frac{\beta}{T} \frac{\partial\langle\mathcal{U}\rangle}{\partial \beta}
$$

so that

$$
\begin{aligned}
\mathcal{C}= & -\frac{1}{Z^{2}}\left[\pi U_{0} \phi\left(\frac{1}{2} ; 2 ; \beta U_{0}\right)+\frac{\pi \beta U_{0}^{2}}{4} \phi\left(\frac{3}{2} ; 3 ; \beta U_{0}\right)\right] \\
& \times\left[\frac{\pi \beta U_{0}}{T} \phi\left(\frac{1}{2} ; 2 ; \beta U_{0}\right)+\frac{\pi \beta^{2} U_{0}^{2}}{4 T} \phi\left(\frac{3}{2} ; 3 ; \beta U_{0}\right)\right] \\
& +\frac{1}{\mathcal{Z}}\left[\frac{\pi \beta U_{0}^{2}}{2 T} \phi\left(\frac{3}{2} ; 3 ; \beta U_{0}\right)+\frac{\pi \beta^{2} U_{0}^{3}}{8 T} \phi\left(\frac{5}{2} ; 4 ; \beta U_{0}\right)\right],
\end{aligned}
$$

depicted in Figure 3. Note that at very low temperatures, quantum effects raise their head and our treatment becomes invalid. Thus, the third thermodynamics' law is violated here. Interestingly enough there is a Schottky anomaly. This is an effect typical of solid-state physics: the specific heat at low temperature exhibits a peak. When $T$ is high, the specific heat decreases. A new effect is observed at very large $T$. The specific heat becomes negative. Such outcome is typical of classical self-gravitating systems [12] [13] [15].

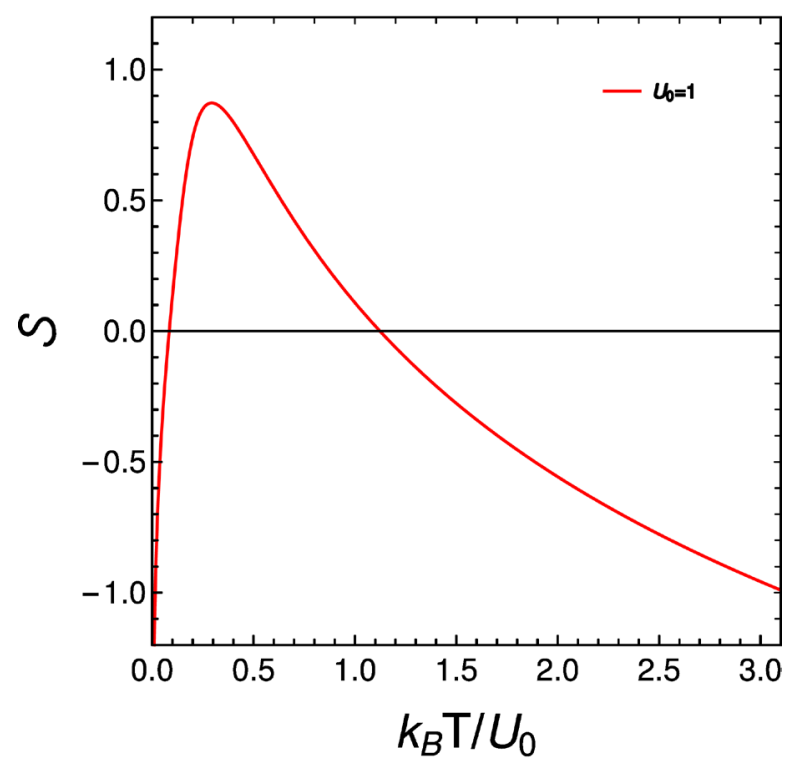

Figure 2. Entropy in appropriate units. Negative values at low $T$ reflect on quantum effects that need to be considered. Those at high $T \geq 10^{22}$ are discussed in the text. 


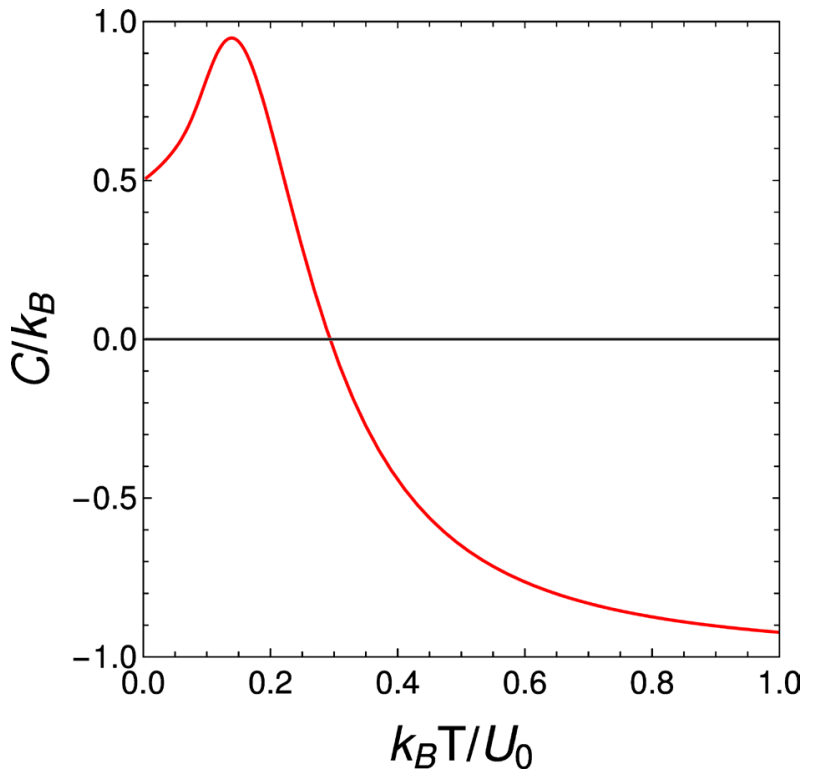

Figure 3. Specific heat in appropriate units. The third thermodynamics' law is violated here because our treatment is classical. A Schottky effect is clearly visible (see text).

\section{Moment Generating Functions}

We pass to the moment generating function for our extant probability distribution function (PDF) $f(x)$ [consult (2.1)]

$$
f(x)=\frac{\mathrm{e}^{\frac{\beta U_{0}}{1+x^{2}}}}{\mathcal{Z}},
$$

where $\mathcal{Z}$ is given by (2.1). In the naive traditional treatment, these moments diverge. The mean value for $x^{2 n+1},(n=1,2,3, \cdots)$ vanishes by parity. That of $x^{2 n}$ becomes

$$
\left\langle x^{2 n}\right\rangle=\frac{1}{\mathcal{Z}} \int_{-\infty}^{\infty} x^{2 n} \mathrm{e}^{\frac{\beta U_{0}}{1+x^{2}}} \mathrm{~d} x .
$$

Appeal again to the variables change $y=1+x^{2}$ and face

$$
\left\langle x^{2 n}\right\rangle=\frac{1}{\mathcal{Z}} \int_{1}^{\infty}(y-1)^{n-\frac{1}{2}} \mathrm{e}^{\frac{\beta U_{0}}{y}} \mathrm{~d} y,
$$

so that, proceeding in a fashion similar to that above we find

$$
\left\langle x^{2 n}\right\rangle=\frac{\beta U_{0}}{\mathcal{Z}} \Gamma\left(-n+\frac{1}{2}\right) \Gamma\left(n+\frac{1}{2}\right) \phi\left(\frac{1}{2}-n ; 2 ; \beta U_{0}\right),
$$

Thus, we get for the moment generating function $\mathcal{M}_{1}(t)$

$$
\mathcal{M}_{1}(t)=\frac{\beta U_{0}}{\mathcal{Z}} \sum_{n=0}^{\infty} \frac{t^{2 n}}{(2 n) !} \Gamma\left(\frac{1}{2}-n\right) \Gamma\left(\frac{1}{2}+n\right) \phi\left(\frac{1}{2}-n ; 2 ; \beta U_{0}\right) .
$$

As particular cases, we obtain the values

$$
\left\langle x^{2}\right\rangle=-\frac{\pi \beta U_{0}}{\mathcal{Z}} \phi\left(-\frac{1}{2} ; 2 ; \beta U_{0}\right),
$$


and

$$
\left\langle x^{4}\right\rangle=\frac{\pi \beta U_{0}}{\mathcal{Z}} \phi\left(-\frac{3}{2} ; 2 ; \beta U_{0}\right)
$$

The first one is plotted in Figure 4. We encounter again here the high temperature effect already reported in [2] [12] [13] (and references therein) and in precedent graphs: a high temperature upper bound, beyond which our treatment becomes invalid. Such bound manifests itself in making negative these types of expectation values at temperatures of the order of $10^{22}$ Kelvin. For reference, 100 seconds after the Big Bang it is estimated that the temperature is of a billion K-degrees, and 0.0001 seconds after the Big Bang it is of about $T=10^{13} \mathrm{~K}[16]$.

\section{Fisher Information Measure (FIM)}

Given a continuous probability distribution function (PDF) $f(x)$ with $x \in \Delta \subset \mathbb{R}$ and $\int_{\Delta} f(x) \mathrm{d} x=1$, its associated Shannon Entropy $\mathcal{S}$ is, as we saw above,

$$
\mathcal{S}(f)=-\int_{\Delta} f \ln (f) \mathrm{d} x
$$

a quantifier of global nature that it is not very sensitive to strong changes in the distribution that may take place in a small-sized region. This is not the case for Fisher's Information Measure (FIM) $\mathcal{F}$ [17] [18], which constitutes a quantifier of the gradient content of $f(x)$, being accordingly quite sensitive even to small localized perturbations. One writes

$$
F(f)=\int_{\Delta} \frac{1}{f(x)}\left[\frac{\mathrm{d} f(x)}{\mathrm{d} x}\right]^{2} \mathrm{~d} x=4 \int_{\Delta}\left[\frac{\mathrm{d} \psi(x)}{\mathrm{d} x}\right]^{2}
$$

FIM can be interpreted in variegated fashions. 1) As a quantifier of the ability

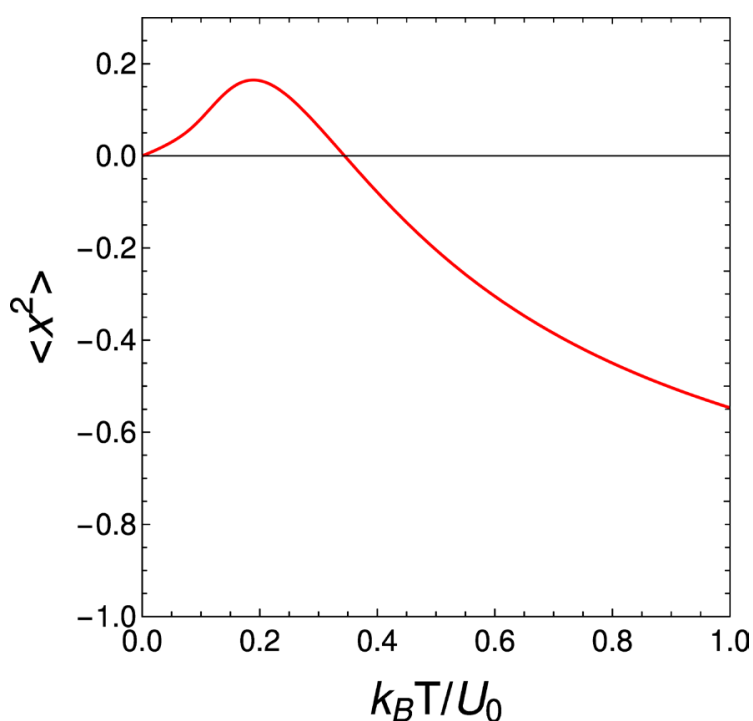

Figure 4. $\left\langle x^{2}\right\rangle$ values in appropriate units (see text). The unphysical negative values emerge at temperatures higher than $10^{22}$ Kelvin. 
to estimate a parameter. 2) As the amount of information that can be extracted from a set of measurements. 3) A quantifier of the state of disorder of a system or phenomenon [18], and finally, at more recent times 4) As a strict measure of order [19] [20] [21]. In the above definition of FIM the division by $f(x)$ is not desirable if $f(x) \rightarrow 0$ at certain $x$-values. We bypass this issue by working with a real probability amplitudes $f(x)=\psi^{2}(x)$ [17] [18], which is a simpler form (no divisors), while showing that $\mathcal{F}$ simply measures the gradient content of $\psi(x)$. The gradient operator significantly influences the contribution of minute local $f$-changes in FIM's values. Thus, this quantifier is called a local measure [18].

For the $f$ of (3.5) one has

$$
\mathcal{F}(f)=\frac{2}{\mathcal{Z}} \int_{0}^{\infty} \mathrm{e}^{\frac{\beta U_{0}}{1+x^{2}}}\left[\frac{\mathrm{de}^{\frac{\beta U_{0}}{1+x^{2}}}}{\mathrm{~d} x}\right]^{2} \mathrm{~d} x,
$$

or

$$
\mathcal{F}(f)=\frac{8 \beta^{2} U_{0}^{2}}{\mathcal{Z}} \int_{0}^{\infty} \frac{x^{2}}{\left(1+x^{2}\right)^{2}} \mathrm{e}^{\frac{\beta U_{0}}{1+x^{2}}} \mathrm{~d} x .
$$

Changing variables in the fashion $y=1+x^{2}$ we get

$$
\mathcal{F}(f)=\frac{4 \beta^{2} U_{0}^{2}}{\mathcal{Z}} \int_{1}^{\infty} y^{-2}(y-1)^{\frac{1}{2}} \mathrm{e}^{\frac{\beta U_{0}}{y}} \mathrm{~d} y,
$$

that after evaluation yields for the Fisher information measure the value

$$
\mathcal{F}(f)=2 \beta U_{0},
$$

clearly a very large positive number, given the smallness of the Boltzmann constant entering the denominator. Let us look for the Cramer-Rao (CR) product $\left[\mathcal{F}(f)\left\langle x^{2}\right\rangle_{f}\right]$, that is always $\geq 1 \quad[18]$. The CR relation has been linked to the Heisenberg uncertainty relation (HUR) for the $D$-dimensional quantum central problem [22]. Still further, Frieden has shown that all UHRs can be derived from the CR relation [18].

We need a value for $\left\langle x^{2}\right\rangle$, that we take from (4.4). The Cramer-Rao product $\left\langle x^{2}\right\rangle \mathcal{F}$ is then

$$
\mathcal{F}(f)\left\langle x^{2}\right\rangle=-\frac{2 \pi \beta^{2} U_{0}^{2}}{\mathcal{Z}} \phi\left(-\frac{1}{2} ; 2 ; \beta U_{0}\right) .
$$

The CR product is plotted in Figure 5. We see that it is indeed $\geq 1$ till we reach a very high temperature, of the order of $10^{22}$ Kelvin, at which our probability distribution no longer makes sense. We have already encountered above this effect, in connection with $\left\langle x^{2}\right\rangle$-graph, the entropy, and the specific heat.

\section{Conclusions}

In deceptively simple fashion, we have regularized the partition function for Brownian functions moving in an external potential, thus solving a very old 


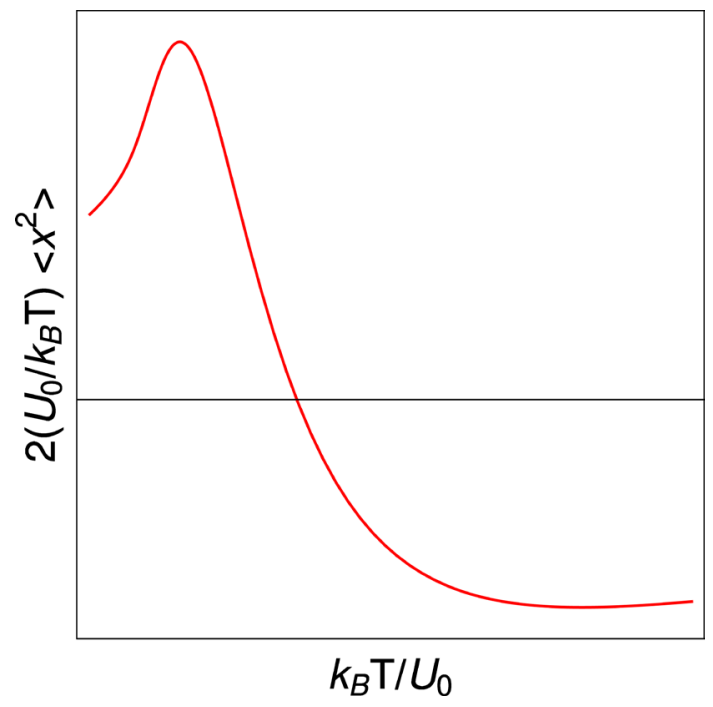

Figure 5. The product of $\left\langle x^{2}\right\rangle$ times Fisher's information measure (Cramer-Rao) (in appropriate units). The well-known associated bound is seen to be violated for temperatures higher than $10^{22}$ Kelvin (see text).

problem. Some other special cases were already treated by the present authors. One is that of the $Z$-expression in the case of Newton's gravity [12], where the divergences are of a different nature from the ones here discussed. A second case is that of Fermi's problem, cited in the Introduction [23]. Our treatment displays two noticeable features.

- Being of a classical nature, it fails at very low temperatures, where quantum effects become predominant.

- At extremely high temperatures, of the order of $10^{22} \mathrm{Kelvin}$, we face a $T$-upper bound. This fact has already been reported, in another context, by Refs. [12] [13]. Our partition function is saying to us that the system can not exist at such high temperatures.

Summing up: We were here tackling partition function' divergences, a physically-motivated mathematical problem, that we indeed solved. As for applications, the most we can say at this stage is that we have at our disposal a new canonical probability distribution. Can one use the concomitant partition function $Z$ in a concrete problem? To answer this question, more research is needed. We guess that with this $Z$ some density distribution might be constructed that could describe a quasi-stationary solution in some suitable scenario.

\section{Conflicts of Interest}

The authors declare no conflicts of interest regarding the publication of this paper.

\section{References}

[1] Fermi, E. (1924) Zeitschrift fur Physik, 26, 54. https://doi.org/10.1007/BF01327311

[2] Plastino, A., Rocca, M.C. and Ferri, G.L. (2019) Physica A, 534, 15. 
https://doi.org/10.1016/j.physa.2019.122169

[3] McQuarrie, D.A. (1975) Statistical Mechanics. Harper and Row, New York.

[4] Defaveri, L., Anteneodo, C., Kessler, D.A. and Barkai, E. (2020) Physical Review Research, 2, Article ID: 043088.

[5] Aghion, E., Kessler, D.A. and Barkai, E. (2019) Physical Review Letters, 122, Article ID: 010601. https://doi.org/10.1103/PhysRevLett.122.010601

[6] Aghion, E., Kessler, D.A. and Barkai, E. (2020) Chaos Solitons \& Fractals, 138, Article ID: 109890.

[7] Ryabov, A., Holubec, V. and Berestneva, E. (2019) Journal of Statistical Mechanics, 2019, Article ID: 084014. https://doi.org/10.1088/1742-5468/ab333f

[8] Siler, M., Ornigotti, L., Brzobohat'y, O., J'akl, P., Ryabov, A., Holubec, V. and Filip, R. (2018) Physical Review Letters, 121, Article ID: 230601. https://doi.org/10.1103/PhysRevLett.121.230601

[9] Neri, I. (2020) Physical Review Letters, 124, Article ID: 040601. https://doi.org/10.1103/PhysRevLett.124.040601

[10] Schwartz, L. (1966) Théorie des distributions. Hermann, Paris.

[11] Gradshteyn, I.S. and Rizhik, I.M. (1965) Table of Integrals Series and Products. Academic Press, Cambridge.

[12] Plastino, A. and Rocca, M.C. (2018) Physica A, 503, 793. https://doi.org/10.1016/j.physa.2018.03.019

[13] Pennini, F., Plastino, A., Rocca, M.C. and Ferri, G.L. (2019) Entropy, 21, 677. https://doi.org/10.3390/e21070677

[14] Bountis, T. and Helleman, R.H.G. (1978) Journal of Mathematical Physics, 19, 477. https://doi.org/10.1063/1.523669

[15] Lynden-Bell, D. and Lynden-Bell, R.M. (1977) Monthly Notices of the Royal Astronomical Society, 181, 405. https://doi.org/10.1093/mnras/181.3.405

[16] Brief History of the Universe. See Web Page. http://www.astro.ucla.edu/wright/BBhistory.html

[17] Fisher, A. (1922) Philosophical Transactions of the Royal Society of London, 222, 309. https://doi.org/10.1098/rsta.1922.0009

[18] Roy Frieden, B. (2004) Science from Fisher information: A Unification. Cambridge University Press, Cambridge. https://doi.org/10.1017/CBO9780511616907

[19] Pennini, F., Plastino, A. and Ferri, G.L. (2012) Entropy, 14, 2081. https://doi.org/10.3390/e14112081

[20] Frieden, B.R. and Hawkins, R.J. (2010) Physical Review E, 82, Article ID: 066117. https://doi.org/10.1103/PhysRevE.82.066117

[21] Frieden, B.R., Plastino, A. and Plastino, A.R. (2011) Physica A, 391, 410. https://doi.org/10.1016/j.physa.2011.08.005

[22] Dehesa, J.S., Gonzalez-Ferez, R. and Sanchez-Moreno, P. (2007) Journal of Physics A: Mathematical and Theoretical, 40, 1845. https://doi.org/10.1088/1751-8113/40/8/011

[23] Plastino, A. and Rocca, M.C. (2019) Physica A, 543, Article ID: 122169. https://doi.org/10.1016/j.physa.2019.122169 